\title{
Characterizations of Integral Type for Weighted Classes of Analytic Banach Function Spaces
}

\author{
Amnah E. Shammaky ${ }^{1}$ and A. El-Sayed Ahmed (iD ${ }^{2}$ \\ ${ }^{1}$ Department of Mathematics, Faculty of Science, Jazan University, Jazan, Saudi Arabia \\ ${ }^{2}$ Mathematics Department, Faculty of Science, Taif University, P.O. Box 11099, Taif 21944, Saudi Arabia
}

Correspondence should be addressed to A. El-Sayed Ahmed; ahsayed80@hotmail.com

Received 6 June 2021; Accepted 12 October 2021; Published 28 January 2022

Academic Editor: V. Ravichandran

Copyright (c) 2022 Amnah E. Shammaky and A. El-Sayed Ahmed. This is an open access article distributed under the Creative Commons Attribution License, which permits unrestricted use, distribution, and reproduction in any medium, provided the original work is properly cited.

The aim of this study is to give some new definitions of Banach spaces of holomorphic functions. Some holomorphic characterizations of integral type for some classes of Banach spaces of holomorphic functions are established in the unit disc $U$.

\section{Introduction and Preliminaries}

Numerous studies of analytic function spaces by several classes of functions are introduced and intensively studied. The theory of function spaces provides interesting tools in many active branches of mathematics, especially in mathematical analysis such as in operator theory, measure theory, and differential equations. In the present study, we aim to give the definition of $\mathbf{g}$-Bloch space of holomorphic functions. Using the new class of functions, some essential relations between it and some other known classes are investigated.

Next, we report the recent advancements of the concepts of specific-weighted classes of holomorphic function spaces. The choice of the appropriate functions gives the specific essential properties of the underlying weighted classes of functions that can have an important impact for the study.

Specific weighted classes of holomorphic function spaces and concepts are presented. Let $U=\{w: w \in C,|w|<1\}$, and $H(U)$ denote the class of all holomorphic functions that belonging to $U$.
The known analytic Bloch-type space [1-5] is defined by

$$
\mathscr{B}=\left\{f: f \in H(U) \text { and } \sup _{w \in U}\left(1-|w|^{2}\right)\left|f^{\prime}(w)\right|<\infty\right\} \text {. }
$$

The analytic little Bloch-type space $\mathscr{B}_{0}$ is symbolized by $\mathscr{B}_{0}$, for which

$$
\mathscr{B}_{0}=\left\{f: f \in H(U) \text { and } \lim _{|w| \longrightarrow 1^{-}}\left(1-|w|^{2}\right)\left|f^{\prime}(w)\right|=0\right\} .
$$

For numerous global studies on Bloch-type spaces, we refer to [6-13] and others.

The analytic Dirichlet-type space $[1,14]$ is given by

$$
\mathscr{D}=\left\{f: f \in H(U) \text { and } \int_{U}\left|f^{\prime}(w)\right|^{2} \mathrm{~d} \sigma_{w}<\infty\right\},
$$

where $\mathrm{d} \sigma_{w}=\mathrm{d} x \mathrm{~d} y$. 
Using Green's function $g\left(w, w_{0}\right)=\ln \mid\left(1-\bar{w}_{0} w\right) /\left(w_{0}-\right.$ $w) \mid=\ln \left(1 /\left|\phi_{w_{0}}(w)\right|\right)$ as a weight function, the analytic $Q_{p}$-spaces is defined in [1] by

$$
\mathbb{Q}_{p}=\left\{f: f: f \in H(U) \text { and } \sup _{w_{0} \in U} \int_{U}\left|f^{\prime}(w)\right|^{2} g^{p}\left(w, w_{0}\right) \mathrm{d} \sigma_{w}<\infty\right\} .
$$

By analytic $Q_{p}$-spaces, some interesting relationships between analytic Dirichlet-type space and the analytic Bloch-type space are obtained [1].

For intensive research on analytic $Q_{p}$ spaces, we may refer to $[1,15-18]$ and others. Also, there are certain specific generalizations of these weighted classes of analytic functions in $C^{n}[12,19,20]$. On the other hand, there are some interesting extensions using quaternion-valued functions setting $([21-24])$.

Hereafter, we set

$$
\varphi_{w_{0}}(w)=\frac{w_{0}-w}{1-\bar{w}_{0} w}, \quad w \neq w_{0}
$$

and set

$$
\varphi_{w_{0}}(w)=C<1, \quad \text { when } w=w_{0}
$$

The modified Green's function is introduced by

$$
\mathbf{g}\left(w, w_{0}\right)=\ln \left|\frac{1-\bar{w}_{0} w}{w_{0}-w}\right|=\ln \frac{1}{\left|\varphi_{w_{0}}(w)\right|}
$$

Motivated by the modified Green's function, the following definitions can be presented.

Definition 1. Let $0<m<\infty$ and $0<n<\infty$. For the function $f \in H(U)$, we define the analytic $\mathbf{g}$-Bloch space $\mathscr{B}_{\mathbf{g}}^{n, m}$ as follows:

$$
\mathscr{B}_{\mathbf{g}}^{n, m}=\left\{f: \sup _{w, w_{0} \in U} \frac{\left(1-|w|^{2}\right)^{n}}{\mathbf{g}^{m}\left(w, w_{0}\right)}\left|f^{\prime}(w)\right|<\infty\right\} .
$$

Furthermore, assume that

$$
\mathscr{B}_{\mathbf{g}}^{n, m}(f)=\sup _{w, w_{0} \in U} \frac{\left(1-|w|^{2}\right)^{n}}{\mathbf{g}^{m}\left(w, w_{0}\right)}\left|f^{\prime}(w)\right| .
$$

Example 1. Let $m \in(0, \infty)$. Suppose that

$$
f(w)=\int_{|w|<1}\left(\log \frac{1}{\left|\varphi_{a}(w)\right|}\right)^{m} \mathrm{~d} w .
$$

It is very obvious that the function $f$ is a $\mathbf{g}$-Bloch function.
Remark 1. Using Definition 1, relationships between analytic Dirichlet-type functions and analytic Bloch functions can be characterized.

When $m=0$ and $0<n=\alpha<\infty$, then we will obtain $\alpha$-Bloch space. The case $m \in(-\infty, 0)$ induces some other types of analytic function spaces with different behaviors and can be studied separately.

The little analytic $\mathbf{g}$-Bloch space $\mathscr{B}_{\mathbf{g}}^{n, m, 0}$ can be considered as a subspace of the analytic $\mathbf{g}$-Bloch space that includes all $f \in \mathscr{B}_{\mathbf{g}}^{n, m}$, with

$$
\lim _{|w| \longrightarrow 1^{-}} \lim _{\left|w_{0}\right| \longrightarrow 1^{-}} \frac{\left(1-|w|^{2}\right)^{n}}{\mathbf{g}^{m}\left(w, w_{0}\right)}\left|f^{\prime}(w)\right|=0 .
$$

Remark 2. The symbol $\mathscr{B}_{\mathbf{g}}^{n, m}(f)$ stands for a seminorm, while the usual norm can be defined as

$$
\|f\|_{\mathscr{B}_{\mathbf{g}}^{n, m}}=|f(0)|+\mathscr{B}_{\mathbf{g}}^{n, m}(f) .
$$

Applying the above norm, the space $\mathscr{B}_{\mathrm{g}}^{n, m}$ is a complete normed space (Banach space).

The next lemma can be applied for some results in this study.

Lemma 1 (See $[14]$ ). Let $0<s<\infty$ and assume that $\left|w_{0}\right|<1$. Then,

$$
\int_{\Gamma_{w}\left|1-\overline{w_{0}} w\right|^{2 s}} \mathrm{~d} \Gamma_{w} \leq \frac{k}{\left(1-\left|w_{0}\right|\right)^{s}},
$$

where $k>0$, and $\Gamma_{w}$ defines the boundary of $U$.

\section{2. g-Bloch Space and Dirichlet Space}

Some essential characterizations between the analytic Dirichlet-type space and the analytic $\mathbf{g}$-Bloch space are given in this section.

Proposition 1. Let $f \in H(U)$ and assume that $\left|w_{0}\right|<1$. Then,

$$
\frac{\left(1-|w|^{2}\right)^{2}}{\mathbf{g}^{m}\left(w, w_{0}\right)}\left|f^{\prime}\left(w_{0}\right)\right|^{2} \leq \frac{4}{\pi R^{2}} \int_{U}\left|f^{\prime}\left(w_{0}\right)\right|^{2} \mathrm{~d} \sigma_{w},
$$

where $0<R<1$. 
Proof. Because the pseudohyperbolic disk can be symbolized by

$$
D\left(w_{0}, R\right)=\left\{w: \rho\left(w, w_{0}\right)=\left|\varphi_{w_{0}}(w)\right|=\left|\frac{w-w_{0}}{1-\bar{w}_{0} w}\right|<R\right\},
$$

where $w_{0}$ is its center and $R$ is its radius, then we infer that

$$
\begin{gathered}
\int_{U}\left|f^{\prime}(w)\right|^{2} \mathrm{~d} \sigma_{w} \geq \int_{D\left(w_{0}, R\right)}\left|f^{\prime}(w)\right|^{2} \mathrm{~d} \sigma_{w} \\
=\pi R^{2}\left(1-\left|w_{0}\right|^{2}\right)^{2}\left|f^{\prime}\left(w_{0}\right)\right|^{2} \geq \pi R^{2}\left(1-\left|w_{0}\right|^{2}\right)^{2}\left|f^{\prime}\left(w_{0}\right)\right|^{2} .
\end{gathered}
$$

Using the definition of the modified Green's function as well as the inequalities,

$$
\begin{aligned}
0 & <\varphi_{w_{0}}\left(w_{0}\right)=C<1, \\
\left(1-\left|\varphi_{w_{0}}(w)\right|^{2}\right) & \leq 2 \mathbf{g}\left(w, w_{0}\right),(\text { see }[25]) .
\end{aligned}
$$

We can obtain

$$
\begin{aligned}
\int_{U}\left|f^{\prime}(w)\right|^{2} \mathrm{~d} \sigma_{w} & \geq \pi R^{2} \frac{\left(1-\left|w_{0}\right|^{2}\right)^{2}}{\left(1-\left|\varphi_{w_{0}}\left(w_{0}\right)\right|^{2}\right)^{2}}\left|f^{\prime}\left(w_{0}\right)\right|^{2} \\
& >2 \pi R^{2} \frac{\left(1-\left|w_{0}\right|^{2}\right)^{2}}{C=\mathbf{g}^{2}\left(w_{0}, w_{0}\right)}\left|f^{\prime}\left(w_{0}\right)\right|^{2} .
\end{aligned}
$$

The proof of Proposition 1 is therefore finished.

Corollary 1. In view of Proposition 1, for $\left|w_{0}\right|<1$, we have the following interesting inclusion:

$$
\mathscr{D} \subset \mathscr{B}_{\mathbf{g}}^{2,2} .
$$

Proposition 2. Let $f \in H(U)$ and assume that $\left|w_{0}\right|<1$. Also, we suppose that $n \in[1, \infty)$ and $m \in(0, \infty)$, then

$$
\frac{\left(1-\left|w_{0}\right|^{2}\right)^{2 n}}{\mathbf{g}^{m}\left(w_{0}, w_{0}\right)}\left|f^{\prime}\left(w_{0}\right)\right|^{2} \leq \frac{2^{m}}{\pi R^{2}} \int_{U}\left|f^{\prime}(w)\right|^{2} \mathrm{~d} \sigma_{w},
$$

where $0<R<1$.

Proof. The proof of Proposition 2 can be obtained as in the proof of Proposition 1, with the following changes:

$$
\begin{aligned}
\int_{U}\left|f^{\prime}(w)\right|^{2} \mathrm{~d} \sigma_{w} & \geq \pi R^{2} \frac{\left(1-\left|w_{0}\right|^{2}\right)^{2}}{\left(1-\left|\varphi_{w_{0}}\left(w_{0}\right)\right|^{2}\right)^{m}}\left|f^{\prime}\left(w_{0}\right)\right|^{2} \\
& \geq \frac{\pi}{2^{m}} R^{2} \frac{\left(1-\left|w_{0}\right|^{2}\right)^{2 n}}{\mathbf{g}^{m}\left(w_{0}, w_{0}\right)}\left|f^{\prime}\left(w_{0}\right)\right|^{2} .
\end{aligned}
$$

\section{Corollary 2. Proposition 2 results that}

$$
\mathscr{D} \subset \mathscr{B}_{\mathrm{g}}^{2 n, m},
$$

with $\left|w_{0}\right|<1, n \in[1, \infty)$, and $m \in(0, \infty)$.

Remark 3. Corollaries 1 and 2 interpret that the analytic Dirichlet-type space can be considered as a subspace of the analytic g-Bloch space when $m=n=2$ as well as for $n \in[1, \infty)$ and $m \in(0, \infty)$, respectively.

Proposition 3. Let $f \in H(U)$ and $f \in \mathscr{B}_{\mathbf{g}}^{n, m}$. Suppose that

$$
I(m, n)=\int_{0}^{1} \frac{(\ln (1 / \mathrm{r}))^{m}}{\left(1-r^{2}\right)^{1-n}} r \mathrm{~d} r<\infty .
$$

Then, for $0<m<\infty$ and $0<n<\infty$ with $m+n>0$, we obtain that

$$
\sup _{w \in U} \int_{U}\left|f^{\prime}(w)\right|^{2} \mathrm{~d} \sigma_{w} \leq(2)^{4-m} \pi I(m, n)\left(\mathscr{B}_{\mathbf{g}}^{n, m}(f)\right)^{2} .
$$

Proof. From the definition of $\mathbf{g}$-Bloch space, we have

$$
\frac{\left(1-\left|w_{0}\right|^{2}\right)^{2 n}}{\mathbf{g}^{m}\left(w_{0}, w_{0}\right)}\left|f^{\prime}(w)\right| \leq \mathscr{B}_{\mathbf{g}}^{n, m}(f) .
$$

By a change of the variables technique, we infer that

$$
\begin{aligned}
\int_{U}\left|f^{\prime}(w)\right|^{2} \mathrm{~d} \sigma_{w} \leq & \left(\mathscr{B}_{\mathbf{g}}^{n, m}(f)\right)^{2} \int_{U} \frac{\left(\ln \left(1 /\left|w_{0}\right|\right)\right)^{m}}{\left(1-\left|\varphi_{w_{0}}\left(w_{0}\right)\right|^{2}\right)^{n}} \\
& \frac{\left(1-\left|\varphi_{w_{0}}\left(w_{0}\right)\right|^{2}\right)^{2}}{\left(1-\left|w_{0}\right|^{2}\right)^{2}} \mathrm{~d} \sigma_{w} .
\end{aligned}
$$

Since,

$$
\left(1-\left|\varphi_{w_{0}}\left(w_{0}\right)\right|^{2}\right)=\frac{\left(1-\left|w_{0}\right|^{2}\right)\left(1-|w|^{2}\right)}{\left|1-\overline{w_{0}} w\right|^{2}} .
$$

Therefore,

$$
\begin{aligned}
\int_{U}\left|f^{\prime}(w)\right|^{2} \mathrm{~d} \sigma_{w} & \leq\left(\mathscr{B}_{\mathbf{g}}^{n, m}(f)\right)^{2} \int_{U} \frac{\left(\ln \left(1 /\left|w_{0}\right|\right)\right)^{m}}{\left(1-\left|\varphi_{w_{0}}\left(w_{0}\right)\right|^{2}\right)^{n}} \mathrm{~d} \sigma_{w} \\
& \leq \pi 2^{4-n}\left(\mathscr{B}_{\mathbf{g}}^{n, m}(f)\right)^{2} \int_{0}^{1} \frac{(\ln (1 / \mathrm{r}))^{m}}{\left(1-r^{2}\right)^{1-n}} r \mathrm{~d} r,
\end{aligned}
$$

which implies that

$$
\int_{U}\left|f^{\prime}(w)\right|^{2} \mathrm{~d} \sigma_{w} \leq\left(\mathscr{B}_{\mathrm{g}}^{n, m}(f)\right)^{2} k_{1},
$$

where $0<k_{1}<\infty$. The proof is therefore established. 
Propositions 2 and 3 result in the following fundamental theorem.

Theorem 1. Let $f \in H(U)$; then, we have equivalence between the following statements:

(i) $\underset{m+n>0}{f \in \mathscr{B}_{\mathbf{g}}^{n, m}}$ with $0 \leq m<\infty$ and $0<n<\infty$ with

(ii) $f \in \mathscr{D}$.
Remark 4. The obtained results in Theorem 1 reflexed the major role of the newly definition of the analytic $\mathbf{g}$-Bloch space which has used to get relations between analytic Dirichlet-type space $\mathscr{D}$ and $\mathscr{B}_{\mathbf{g}}^{n, m}$ space.

\section{3. $g$-Bloch Functions and $Q_{p}$ Functions}

Proposition 4. For $0 \leq m<\infty, 1 \leq n<\infty$, let $f \in H(U)$ and $\left|w_{0}\right|<1$; hence,

$$
\frac{\left(1-\left|w_{0}\right|^{2}\right)^{2 n}}{\mathbf{g}^{m}\left(w_{0}, w_{0}\right)}\left|f^{\prime}\left(w_{0}\right)\right|^{2} \leq \frac{2^{2 m+4}}{\rho^{2}\left(1-\rho^{2}\right)^{p}} \int_{U}\left|f^{\prime}(w)\right|^{2}\left(1-\left|\varphi_{w_{0}}(w)\right|^{2}\right)^{p} \mathrm{~d} \sigma_{w},
$$

where $0<\rho<1$ and $p \in(0, \infty)$.

Proof. From subharmonicity principle, the following inequality can be easily obtained:

$$
|h(0)|^{2} \leq \frac{1}{\rho^{2}} \int_{D(0, \rho)}|h(z)|^{2} \mathrm{~d} \sigma_{w}
$$

Since $f \in H(U)$ is holomorphic on $D\left(w_{0}, \rho\right)$, then use (31) to the function

$$
h=f^{\prime} \circ \varphi_{w_{0}} .
$$

Applying the technique of change of variables, the next inequality can be inferred:

$$
\begin{aligned}
\left|f^{\prime}\left(w_{0}\right)\right|^{2} & \leq \frac{1}{\rho^{2}} \int_{D(0, \rho)}\left|f^{\prime}\left(\varphi_{w_{0}}(w)\right)\right|^{2} \mathrm{~d} \sigma_{w} \\
& =\frac{1}{\rho^{2}} \int_{D\left(w_{0}, \rho\right)}\left|f^{\prime}(w)\right|^{2}\left(\frac{1-\left|\varphi_{w_{0}}(w)\right|^{2}}{1-|w|^{2}}\right)^{2} \mathrm{~d} \sigma_{w} .
\end{aligned}
$$

From [5], we have

$$
\left(\frac{1-\left|\varphi_{w_{0}}(w)\right|^{2}}{1-|w|^{2}}\right)^{2} \leq\left(\frac{4}{\left(1-\left|w_{0}\right|^{2}\right)}\right)^{2}
$$

This yields that

$$
\left|f^{\prime}\left(w_{0}\right)\right|^{2} \leq \frac{(4 / \rho)^{2}}{\left(1-\left|w_{0}\right|^{2}\right)^{2}} \int_{D\left(w_{0}, \rho\right)}\left|f^{\prime}(w)\right|^{2} \mathrm{~d} \sigma_{w}
$$

Because,

$$
\begin{aligned}
\left(1-\left|\varphi_{w_{0}}(w)\right|^{2}\right)^{p} & \geq\left(1-\rho^{2}\right)^{p}, \\
\varphi_{w_{0}}\left(w_{0}\right) & =0 .
\end{aligned}
$$

Then,

$$
\begin{aligned}
\int_{D\left(w_{0}, \rho\right)}\left|f^{\prime}(w)\right|^{2}\left(1-\left|\varphi_{w_{0}}(w)\right|^{2}\right)^{p} \mathrm{~d} \sigma_{w} & \geq \frac{\rho^{2}\left(1-\rho^{2}\right)^{p}}{16}\left(1-\left|w_{0}\right|^{2}\right)^{2}\left|f^{\prime}\left(w_{0}\right)\right|^{2} \\
& \geq \frac{\rho^{2}\left(1-\rho^{2}\right)^{p}}{16} \frac{\left(1-\left|w_{0}\right|^{2}\right)^{2}}{\left(1-\left|\varphi_{w_{0}}\left(w_{0}\right)\right|^{2}\right)^{2}}\left|f^{\prime}\left(w_{0}\right)\right|^{2} \\
& \geq \frac{\rho^{2}\left(1-\rho^{2}\right)^{p}}{2^{2 m+4}} \frac{\left(1-\left|w_{0}\right|^{2}\right)^{2 n}}{\mathbf{g}^{2 m}\left(w, w_{0}\right)}\left|f^{\prime}\left(w_{0}\right)\right|^{2} .
\end{aligned}
$$

Using the inequality 


$$
\int_{U}\left|f^{\prime}(w)\right|^{2}\left(1-\left|\varphi_{w_{0}}(w)\right|^{2}\right)^{p} \mathrm{~d} \sigma_{w} \geq \int_{D\left(w_{0}, \rho\right)}\left|f^{\prime}(w)\right|^{2}\left(1-\left|\varphi_{w_{0}}\left(w_{0}\right)\right|^{2}\right)^{p} \mathrm{~d} \sigma_{w}
$$

we can deduce that

$$
\frac{\left(1-\left|w_{0}\right|^{2}\right)^{2 n}}{\mathbf{g}^{2 m}\left(w_{0}, w\right)}\left|f^{\prime}\left(w_{0}\right)\right|^{2} \leq \frac{2^{2 m+4}}{\rho^{2}\left(1-\rho^{2}\right)^{p}} \int_{U}\left|f^{\prime}(w)\right|^{2}\left(1-\left|\varphi_{w_{0}}(w)\right|^{2}\right)^{p} \mathrm{~d} \sigma_{w}
$$

The proof is therefore completely finished.

$$
\mathscr{B}_{\mathbf{g}}^{2 n, 2 m}(f) \leq \frac{2^{2 m+4}}{\rho^{2}\left(1-\rho^{2}\right)^{p}} Q_{p}^{2}(f),
$$

Corollary 3. By Proposition 4, for $p>0,0 \leq m<\infty$, $1 \leq n<\infty$, and $\left|w_{0}\right|<1$, the following inclusions can be easily

where proved:

$$
Q_{p}(f)=\sup _{w_{0} \in U} \int_{U}\left|f^{\prime}(w)\right|^{2}\left(1-\left|\varphi_{w_{0}}(w)\right|^{2}\right)^{p} \mathrm{~d} \sigma_{z} \approx \sup _{w_{0} \in U} \int_{U}\left|f^{\prime}(w)\right|^{2}\left(\mathbf{g}\left(w, w_{0}\right)\right)^{p} \mathrm{~d} \sigma_{w}<\infty .
$$

Proposition 5. Let $f \in H(U)$. Assume that $0 \leq m<\infty$ and $0<n<\infty$ with $m+n+p>0$. Then, we have that

$$
\int_{U}\left|f^{\prime}(w)\right|^{2}\left(\mathbf{g}\left(w, w_{0}\right)\right)^{p} \mathrm{~d} \sigma_{w} \leq I(m, n, p)\left(\mathscr{B}_{\mathbf{g}}^{m, n}\right)^{2}(f)
$$

$$
I(m, n, p)=\lambda \int_{0}^{1} \frac{(\ln (1 / \mathrm{r}))^{m+p}}{(1-r)^{1-n}} r \mathrm{~d} r
$$

and $\lambda$ is an absolute positive constant.

(42) Proof. It is not hard to see that

where

$$
\int_{U}\left|f^{\prime}(w)\right|^{2} \mathbf{g}^{p}\left(w_{0}, w\right) \mathrm{d} \sigma_{w} \leq 4\left(\mathscr{B}_{\mathbf{g}}^{m, n}\right)^{2}(f) \int_{U} \frac{(\ln (1 /|w|))^{m+p}}{\left(1-|w|^{2}\right)^{2-2 n}} \mathrm{~d} \sigma_{w}
$$

Applying Lemma 1, we obtain

$$
\begin{aligned}
& \int_{U}\left|f^{\prime}(w)\right|^{2} \mathbf{g}^{p}\left(w_{0}, w\right) \mathrm{d} \sigma_{w} \leq 8 \pi\left(\mathscr{B}_{\mathbf{g}}^{m, n}\right)^{2}(f) \int_{0}^{1} \frac{(\ln (1 / \mathrm{r}))^{m+p}}{(1-r)^{1-n}} r \mathrm{~d} r \\
& =I(m, n, p)\left(\mathscr{B}_{\mathbf{g}}^{m, n}\right)^{2}(f) .
\end{aligned}
$$

Combining Corollary 3 and Proposition 5, we have the following theorem:

Theorem 2. Let $h \in H(U)$. Assume that $0 \leq m<\infty$ and $0<n<\infty$ with $m+n+p>0$ as well as $0<p<\infty$. Therefore, the next assertions can be equivalent:

(a) $h \in \mathscr{B}_{\mathbf{g}}^{m, n}$ (b) $h \in \mathbb{Q}_{p}$

(c) $h \in \mathbb{Q}_{p}$ for some $0<p<\infty$.

Proof. $(a) \Rightarrow(b)$ can be established using Proposition 5 . $(b) \Rightarrow(c)$ is quite obvious. $(c) \Rightarrow(a)$ can be proved by Corollary 3. 
Remark 5. Theorem 2 investigates relations between the new type of holomorphic $g$-Bloch functions and the weighted holomorphic Dirichlet-type functions.

\section{A Specific Criteria}

The following symbol stands for a non-Euclidean distance of hyperbolic-type between the points $w_{0}$ and $w$ in $U$.

$$
d\left(w_{0}, w\right)=\ln \sqrt{\mid \frac{\left|1-\overline{w_{0}} w\right|+\left|w_{0}-w\right|}{\left|1-\overline{w_{0}} w\right|-\left|w_{0}-w\right|}}(\text { see [26]). }
$$

Now, for $0<R<\infty$, set

$$
\begin{aligned}
T(w, R) & =\left\{w_{0} \in U: d\left(w_{0}, w\right)<R\right\}, \\
T_{1}(w, R) & =\left\{w_{0} \in U: d\left(w_{0}, w\right)=R\right\} .
\end{aligned}
$$

Let $h \in H(U)$ be the nonconstant analytic function, and let $S_{h}(w, R)$ define the concerned area of the Riemannian image $P(w, R)$ of $T(w, R)$ by the function $h$, and assume that $\mathcal{S}_{h}^{*}(w, R)$ defines the area of the image $P(w, R)$ of $T(w, R)$ using the function $h$. It should be noted that $P(w, R)$ defines the projection to $C$. The length of the Riemannian image of
$T_{1}(w, R)$ by $h$ is denoted by $M_{h}(z, \rho)$, and the symbol $\mathscr{M}_{h}(z, \rho)$ defines the length of the outer boundary of $P(w, R)$. If $\Gamma$ defines a specific bounded domain in $C$, the concept of outer boundary means that we are working in the boundary of $C \backslash B$, where $B$ may be defined as the unbounded component for the complement $C \backslash \Gamma$.

Now, we clearly have the following inequalities:

$$
\begin{gathered}
S_{h}(w, R) \geq \mathcal{S}_{h}^{*}(w, R), \\
M_{h}(w, R) \geq \mathscr{M}_{h}(w, R),
\end{gathered}
$$

where $0<R<\infty$ and each $w \in U$.

Proposition 6. Let $h \in H(U)$ be a nonconstant analytic function. Suppose that $1 \leq n<\infty$ and $0 \leq m<\infty$, then the following inequalities can be deduced:

$$
\frac{\left(1-|w|^{2}\right)^{n}}{\mathbf{g}^{m}\left(w, w_{0}\right)}\left|h^{\prime}(w)\right| \leq \frac{1}{\theta(R)} \sqrt{\frac{2^{m} \mathcal{S}_{h}^{*}(w, R)}{\pi\left(1-\rho^{2}\right)^{m}}} .
$$

Also,

$$
\frac{\left(1-|w|^{2}\right)^{n}}{\mathbf{g}^{m}\left(w, w_{0}\right)}\left|h^{\prime}(w)\right| \leq \frac{2^{m}\left(1-|w|^{2}\right)^{n}}{\left(1-\left|\varphi_{w_{0}}(w)\right|^{2}\right)^{m}}\left|h^{\prime}(w)\right| \leq \frac{2^{m-1} \mathscr{M}_{h}(w, R)}{\left(1-\rho^{2}\right)^{m} \pi \theta(R)}
$$

where $\theta(R)=\left(\left(e^{2 R}-1\right) /\left(e^{2 R}+1\right)\right)$.

Proof. First, we suppose that $h^{\prime}(w) \neq 0$. Now, we suppose that

$$
f\left(w_{0}\right)=h\left(\frac{w_{0}+w}{1+\bar{w} w_{0}}\right)=b_{0}+b_{1} w_{0}+b_{2} w_{0}^{2}+b_{3} w_{0}^{3}+\cdots,
$$

with $\left|w_{0}\right|<1$, where $b_{0}, b_{1}, b_{2}, b_{3}, \ldots$ are the positive constants. Now,

$$
\left(1-|w|^{2}\right)^{n}\left|h^{\prime}(w)\right| \leq b_{1}=\left(1-|w|^{2}\right)\left|h^{\prime}(w)\right| \neq 0,
$$

which implies that

$$
\frac{\left(1-|w|^{2}\right)^{n}}{\mathbf{g}^{m}\left(w, w_{0}\right)}\left|h^{\prime}(w)\right| \leq \frac{\left(1-|w|^{2}\right)}{\mathbf{g}^{m}\left(w, w_{0}\right)}\left|h^{\prime}(w)\right| \neq 0<\infty .
$$

Using Theorems 1 and 2 in [27], the following inequalities can be deduced:

$$
\begin{gathered}
\pi \theta^{2}(R)\left|b_{1}\right|^{2} \leq \mathcal{S}_{h}^{*}(w, R), \\
2 \pi \theta(R)\left|b_{1}\right| \leq \mathscr{M}_{h}(w, R) .
\end{gathered}
$$

Since, $0<\left|\varphi_{w_{0}}(w)\right|<\rho<1$, then

$$
\frac{\left(1-|w|^{2}\right)^{n}}{\mathbf{g}^{m}\left(w, w_{0}\right)}\left|h^{\prime}(w)\right| \leq \frac{1}{\theta(R)} \sqrt{\frac{2^{m} \mathcal{S}_{h}(w, R)}{\pi\left(1-\rho^{2}\right)^{m}}}
$$

Also,

$$
\frac{\left(1-|w|^{2}\right)^{n}}{\mathbf{g}^{m}\left(w, w_{0}\right)}\left|h^{\prime}(w)\right| \leq \frac{2^{m}\left(1-|w|^{2}\right)^{n}}{\left(1-\left|\varphi_{w_{0}}(w)\right|^{2}\right)^{m}}\left|h^{\prime}(w)\right| \leq \frac{2^{m-1} \mathscr{M}_{h}(w, R)}{\left(1-\rho^{2}\right)^{m} \pi \theta(R)} .
$$

Remark 6. In the proof of Proposition 6, positive coefficients are considered to keep the convergence of Taylor or Fourier power series in its region.
Theorem 3. Let $h \in H(U)$ be the nonconstant analytic function. Suppose that $1 \leq n<\infty$ and $0 \leq m<\infty$; therefore, the equivalence between next assertions can be mutually obtained: 
(1) The function $h \in \mathscr{B}_{\mathbf{g}}^{n, m}$

(2) The constant $0<R<\infty$ can be obtained, for which

$$
\sup _{w_{0} \in U} S_{h}(w, R)<\infty
$$

(3) The constant $0<R<\infty$ can be obtained, for which

$$
\sup _{w_{0} \in U} \mathcal{S}_{h}^{*}(w, R)<\infty \text {. }
$$

(4) The constant $0<R<\infty$ can be obtained, for which

$$
\sup _{w_{0} \in U} M_{h}(w, R)<\infty \text {. }
$$

(5) The constant $0<R<\infty$ can be obtained, for which

$$
\sup _{w_{0} \in U} \mathscr{M}_{h}(w, R)<\infty .
$$

Proof. The assertions (2) $\Rightarrow(3)$ and $(4) \Rightarrow(5)$ can be deduced clearly. Additionally, $(1) \Rightarrow(2)$ and $(1) \Rightarrow(4)$ are obvious. Next, suppose that (1) with

$$
\sup _{w_{0} \in U} \frac{\left(1-|w|^{2}\right)^{n}}{\mathbf{g}^{m}\left(w, w_{0}\right)} h^{\prime}(w)=\mathscr{B}_{\mathbf{g}}^{n, m}(h)<\infty .
$$

Hence,

$$
\begin{aligned}
S_{h}(w, R) & =\int_{T(w, R)}\left|h^{\prime}\left(w_{0}\right)\right|^{2} \mathrm{~d} \sigma_{w_{0}} \\
& \leq\left(\mathscr{B}_{\mathbf{g}}^{n, m}(h)\right)^{2} \int_{\left|w_{0}\right|<\theta(R)} \frac{\mathbf{g}^{m}\left(w, w_{0}\right)}{\left(1-|w|^{2}\right)^{n}} \mathrm{~d} \sigma_{w_{0}} \\
& =\eta_{1}(R)\left(\mathscr{B}_{\mathbf{g}}^{n, m}(h),\right.
\end{aligned}
$$

where $\eta_{1}(R)$ is a positive constant depending on $R$. In addition, using the same way, we deduce that

$$
\begin{aligned}
\frac{\left(\mathscr{B}_{\mathbf{g}}^{n, m}(h)\right)^{2}(\theta(R))^{2 n}}{\left(1-(\theta(R))^{2}\right)^{2 m}} & \left.=M_{h}(w, R)=\eta(R)\right) \int_{T_{1}(w, R)}\left|f^{\prime}\left(w_{0}\right)\right| \mathrm{d} \sigma_{w_{0}} \\
& \leq \mathscr{B}_{\mathbf{g}}^{n, m}(h) \int_{T_{1}(w, R)} \frac{\mathbf{g}^{m}\left(w, w_{0}\right)}{\left(1-|w|^{2}\right)^{n}} \mathrm{~d} \sigma_{w_{0}} \\
& =\eta_{2}(R) \mathscr{B}_{\mathbf{g}}^{n, m}(h),
\end{aligned}
$$

where $\eta(R)$ and $\eta_{2}(R)$ are the positive constants depending on $R$.

Additionally, the assertion $(3) \Rightarrow(1)$ as well as $(5) \Rightarrow(1)$ can be proved in view of Proposition 6 .

Remark 7. Theorem 3 gives an interesting and global criterion for analytic g-Bloch-type function by the help of the concrete area as well as the concerned length of the images of both non-Euclidean unified discs and unified circles, respectively. The obtained results in this section extended and improved some specific results in the study by Yamashita [26].

Remark 8. Quite recently, a new study of bicomplex functions was introduced in [28].

An interesting question can be formulated for the newly defined g-Bloch functions as follows. Can we discuss and study the new defined class of analytic g-Bloch type functions in the case of bicomplex functions?

\section{Conclusions}

Function spaces theory is developed, extended, and generalized to spaces of several complex variables $([8-13,29])$ also using quaternion-valued functions ([21-24, 30-33]). The intention of this study is to introduce a new type of analytic function spaces, which plays an interesting and global rule of studying complex function spaces. It should be emphasized that both the worked plane of the study (i.e., $U$ ) and the considered holomorphic functions of Bloch-type as well as specific properties of Green's function are extremely needed for the new classes.

The holomorphic classes of $\mathbf{g}$-Bloch functions are defined and deeply considered using a modified Green's function. By the new function classes, some specific relations and inclusions for the holomorphic Dirichlet-type space as well as holomorphic $Q_{p}$ spaces are obtained. On the other hand, an extension of Yamashita's result [26] is presented using holomorphic g-Bloch functions.

\section{Data Availability}

No data were used to support this study.

\section{Conflicts of Interest}

The authors declare that they have no conflicts of interest.

\section{Acknowledgments}

The authors are grateful to Taif University Researchers concerning the support of project number (TURSP-2020/ 159), Taif University, Saudi Arabia.

\section{References}

[1] R. Aulaskari and P. Lappan, "Criteria for an analytic function to be bloch and a harmonic or meromorphic function to be normal, complex analysis and its applications," Pitman Research Notes in Mathematics Series, vol. 305, pp. 136-146, 1994.

[2] R. Aulaskari, J. Xiao, and R. Zhao, "On subspaces and subsets of BMOA and UBC," Analysis, vol. 15, no. 2, pp. 101-122, 1995.

[3] E. G. Kwon, "A characterization of Bloch space and Besov space," Journal of Mathematical Analysis and Applications, vol. 324, no. 2, pp. 1429-1437, 2006.

[4] M. Pavlovic, "On the Holland-Walsh characterization of Bloch functions," vol. 51, no. 2, pp. 439-441, 2008. 
[5] K. Stroethoff, "Besov-type characterisations for the Bloch space," Bulletin of the Australian Mathematical Society, vol. 39, no. 3, pp. 405-420, 1989.

[6] M. Huang, S. Ponnusamy, and J. Qiao, "Extreme points and support points of harmonic $\alpha$-Bloch mappings," Rocky Mountain Journal of Mathematics, vol. 50, no. 4, pp. 13231354, 2020.

[7] S. Li and S. Stević, "Some characterizations of the Besov space and the $\alpha$-Bloch space," Journal of Mathematical Analysis and Applications, vol. 346, no. 1, pp. 262-273, 2008.

[8] S. Li and H. Wulan, "Besov space on the unit ball of $\mathbb{C}^{n}$," Indian Journal of Mathematics, vol. 48, no. 2, pp. 177-186, 2006.

[9] S. Li and H. Wulan, "Characterizations of $\alpha$-Bloch spaces on the unit ball," Journal of Mathematical Analysis and Applications, vol. 343, no. 1, pp. 58-63, 2008.

[10] Z. Lou and H. Wulan, "Characterisations of Bloch functions in the unit ball of $\mathbb{C}^{n}$," Bulletin of the Australian Mathematical Society, vol. 68, no. No. 2, pp. 205-212, 2003.

[11] M. Nowak, "Bloch space and Möbius invariant Besov spaces on the unit ball of $\mathbb{C}^{n}$," Complex Variables, Theory Applications, vol. 44, no. 1, pp. 1-12, 2001.

[12] C. Ouyang, W. Yang, and R. Zhao, "Möbius invariant $\mathbb{Q}_{p}$ spaces associated with the green's function on the unit ball of $C^{n}$, , Pacific Journal of Mathematics, vol. 1, p. 182, 1998.

[13] R. Zhao, "A characterization of Bloch-type spaces on the unit ball of $\mathbb{C}^{n}$," Journal of Mathematical Analysis and Applications, vol. 330, no. 1, pp. 291-297, 2007.

[14] K. Zhu, Operator Theory in Function Spaces Mathematical Surveys and Monographs 138, American Mathematical Society (AMS), Providence, RI, USA, 2nd edition, 2007.

[15] R. Aulaskari, D. Girela, and H. Wulan, "Taylor coefficients and mean growth of the derivative of $Q_{p}$ functions," Journal of Mathematical Analysis and Applications, vol. 258, no. 2, pp. 415-428, 2001.

[16] R. Aulaskari and L. M. Tovar, "On the function spaces $B^{q}$ and Qp," Bulletin Hong Kong Mathematical Society, vol. 1, pp. 203-208, 1997.

[17] M. Essen and J. Xiao, "spaces $\mathbb{Q}_{p}$-a survey, complex function spaces, proceedings of the summer school, mekrijrvi, Finland," vol. 4, pp. 11-60, 2001.

[18] J. Xiao, Holomorphic Q Classes, Lecture Notes in Mathematics, Springer, Berlin, Germany, 2001.

[19] M. Essen, S. Janson, L. Peng, and J. Xiao, "spaces of several real variables Q," Indiana University Mathematics Journal, vol. 49, no. 2, pp. 575-615, 2000.

[20] S. Feng, "On Dirichlet type spaces, $\alpha$-Bloch spaces and $\mathbb{Q}_{p}$ spaces on the unit ball of $\mathbb{C}^{n}$," Analysis München, vol. 21, no. 1, pp. 41-52, 2001.

[21] K. Gürlebeck and A. El-Sayed Ahmed, "Integral norms for hyperholomorphic Bloch-functions in the unit ball of $\mathbb{R}^{3}$," in Progress in Analysis, Begehr, Ed., vol. 1, pp. 253-263, Kluwer Academic Publishers, London, UK, 2003.

[22] K. Gürlebeck and A. E.-S. Ahmed, "On B Q spaces of hyperholomorphic functions and the Bloch space in $\mathbb{R}^{3}$," in Finite or Infinite Complex Analysis and Its Applications, Advances in Complex Analysis and Applications, L. H. Son, Ed., Kluwer Academic Publishers, Boston, MA, USA, pp. 269-286, 2004.

[23] K. Gürlebeck, U. Kähler, M. V. Shapiro, and L. M. Tovar, "On Qp-spaces of quaternion-valued functions," Complex Variables, Theory and Application: An International Journal, vol. 39, no. 2, pp. 115-135, 1999.
[24] A. El-Sayed Ahmed and S. Omran, "Extreme points and some quaternion valued functions in the unit ball of $\mathbb{R}^{3}$," Advances in Applied Clifford Algebras, vol. 28, no. 1, p. 31, 2018.

[25] R. Aulaskari, M. Nowak, and R. Zhao, "The n-th derivative characterisation of Möbius invariant Dirichlet space," Bulletin of the Australian Mathematical Society, vol. 58, no. 1, pp. 43-56, 1998.

[26] S. Yamashita, "Criteria for functions to be Bloch," Bulletin of the Australian Mathematical Society, vol. 21, no. 2, pp. 223227, 1980.

[27] H. Thomas and H. Macgreogor, "Lenth and area estimates for analytic functions," Michigan Mathematical Journal, vol. 11, pp. 317-320, 1964.

[28] L. F. Tovar and L. M. Tovar, "Bicomplex bergman and Bloch spaces," Arabian Journal of Mathematics, vol. 9, no. 3, pp. 665-679, 2020.

[29] A. Miralles, "Bloch functions on the unit ball of a Banach space," Proceedings of the American Mathematical Society, vol. 149, no. 4, pp. 1459-1470, 2021.

[30] A. El-Sayed Ahmed, "Lacunary series in weighted hyperholomorphic $B^{p, q}(G)$ spaces," Numerical Functional Analysis and Optimization, vol. 32, no. 1, pp. 41-58, 2011.

[31] A. El-Sayed Ahmed, "Hyperholomorphic Q classes," Mathematical and Computer Modelling, vol. 55, no. 3-4, pp. 1428-1435, 2012.

[32] A. El-Sayed Ahmed, K. Gürlebeck, L. F. Tovar, and L. M. Tovar, "Characterizations for Bloch space by $B^{p, q}$ spaces in clifford analysis," Complex Variables and Elliptic Equations, vol. 51, no. 2, pp. 119-136, 2006.

[33] S. G. Gal and I. Sabadini, "Polynomial approximation in quaternionic Bloch and Besov spaces," Advances in Applied Clifford Algebras, vol. 30, no. 5, p. 64, 2020. 